\title{
Análise clínica e radiológica do resultado placa ponte versus fixador externo na fratura cominutiva do rádio distal*
}

\section{Analysis of the Clinical and Radiological Results of Bridge Plate Versus External Fixation in Comminuted Distal Radius Fractures}

\author{
Beatriz Canhoto Carula10 Matheus da Silva Pereira ${ }^{1}$ Ana Paula Bonilauri Ferreira ${ }^{2}$ (1) \\ Henrique Ayzemberg ${ }^{3}$ Valdir Steglich ${ }^{3}$ Tiago Salati Stangarlin ${ }^{3}$ \\ ${ }^{1}$ Instituto de Ortopedia e Traumatologia de Joinville, Hospital \\ Municipal São José, Joinville, SC, Brasil \\ ${ }^{2}$ Curso de Odontologia, Universidade da Região de Joinville \\ (UNIVILLE), Joinville, SC, Brasil \\ ${ }^{3}$ Serviço de Residência Médica, Instituto de Ortopedia e Traumatologia de \\ Joinville, Hospital Municipal São José, Joinville, SC, Brasil \\ Rev Bras Ortop 2021;56(1):61-68. \\ Endereço para correspondência Ana Paula Bonilauri Ferreira, PhD, \\ Rua Ex-Combatentes, 125, Joinville, SC, 89221-103, Brasil \\ (e-mail: apbonilauri@gmail.com).
}

\section{Resumo}

Palavras-chave

- rádio distal

- placas ósseas

- fixadores externos
Objetivo Avaliar e comparar os resultados clínicos e radiológicos de pacientes com fraturas cominutivas distais do rádio tratados com fixador externo ou placa ponte dorsal. Métodos Foram analisados 45 pacientes, sendo 18 tratados com fixador externo, e 25, com placa ponte dorsal, após 1 ano de pós-operatório. Aplicou-se uma escala analógica de dor e o questionário Disabilities of the Arm, Shouder and Hand (DASH), além de análise radiográfica, da avaliação de força, e da amplitude de movimento. As análises estatísticas foram realizadas utilizando o teste qui-quadrado e o teste não paramétrico de Mann-Whitney.

Resultados A fratura foi mais comum em mulheres acima de 60 anos por queda do mesmo nível. Ambos os métodos demonstraram resultados funcional e radiológico similares. A infecção foi mais prevalente com o uso do fixador externo, mas a força de preensão residual foi melhor. Neuropatia simpático-reflexa foi mais comum com o uso da placa ponte dorsal.

Conclusão Não houve consenso da superioridade de um método em relação ao outro em nossa análise. Cada um dos métodos apresenta vantagens e desvantagens, mas ambos mostraram resultados bons e semelhantes. A escolha do tratamento deve ser atribuída ao perfil do trauma, às condições clínicas do paciente, à experiência do profissional, e à disponibilidade de materiais.
Trabalho desenvolvido no Instituto de Ortopedia e Traumatologia de Joinville, Hospital Municipal São José, Joinville, SC, Brasil. recebido

16 de Agosto de 2019

aceito

15 de Abril de 2020

Publicado online

Setembro 24, 2020
DOI https://doi.org/

10.1055/s-0040-1713393. ISSN 0102-3616.
(C) 2020. Sociedade Brasileira de Ortopedia e Traumatologia. All rights reserved.

This is an open access article published by Thieme under the terms of the Creative Commons Attribution-NonDerivative-NonCommercial-License, permitting copying and reproduction so long as the original work is given appropriate credit. Contents may not be used for commercial purposes, or adapted, remixed, transformed or built upon. (https://creativecommons.org/ licenses/by-nc-nd/4.0/)

Thieme Revinter Publicações Ltda., Rua do Matoso 170, Rio de Janeiro, RJ, CEP 20270-135, Brazil 


\begin{abstract}
Keywords

- distal radius

- bone plates

- external fixator

Objective To evaluate and compare the clinical and radiological outcomes of patients with comminuted distal radius fractures treated with an external fixator or a dorsal bridge plate.

Methods In total, 45 patients were analyzed 1 year after surgery; 18 were treated with an external fixator, and 25 received a dorsal bridge plate. An analog pain scale and the Disabilities of the Arm, Shoulder and Hand (DASH) questionnaire were applied, in addition to radiographic, strength and range of motion assessments. Statistical analyzes were performed using the chi-squared test and the Mann-Whitney nonparametric test.

Results Fractures were more common in women over 60 years old who suffered falls from their own height. Both methods demonstrated similar functional and radiological results. Infections were more prevalent in patients receiving external fixators, but their residual grip strength was better. Reflex sympathetic neuropathy was more common in subjects treated with a dorsal bridge plate.

Conclusion Our analysis showed no consensus on the superiority of one method over the other. Each method had advantages and disadvantages, but both led to good, similar outcomes. The treatment must be chosen according to the profile of the trauma, the patient's clinical conditions, the surgeon's experience, and the availability of materials.
\end{abstract}

\section{Introdução}

As fraturas distais do rádio correspondem a $12 \%$ das fraturas em adultos, sendo grande causa de morbidade. ${ }^{1}$ Na população mais jovem, ocorrem por traumas de alta energia, com fraturas mais graves e cominutas, e, na população idosa, por trauma de baixa energia. ${ }^{2}$ Essas fraturas, quando não recebem o tratamento adequado, resultam em dor crônica, limitação do movimento, e prejuízo funcional, ${ }^{3}$ tendo grande impacto na qualidade de vida do paciente, e trazendo consequências para a saúde pública e onerando o sistema, visto que grande parte são pacientes jovens que ficam incapacitados para o trabalho. ${ }^{2}$

Apesar da grande prevalência e das consequências sociais dessa fratura, ainda não há consenso de qual é o melhor tratamento, devido à grande possibilidade de perfis de fraturas distais do rádio. ${ }^{4}$ Para avaliar a melhor forma de tratamento, deve-se avaliar o padrão da fratura (classificação, qualidade óssea), o perfil do paciente (idade, atividades diárias), a associação com outras lesões (ligamentares ou ósseas), e a experiência do cirurgião com os materiais. ${ }^{5}$

As fraturas distais do rádio podem ser tratadas tanto de forma conservadora quanto cirúrgica, dependendo dos fatores citados anteriormente. ${ }^{6}$ Nenhum tratamento é isento de complicações, devendo ser indicado individualmente para cada paciente. $O$ tratamento cirúrgico pode ser benéfico em fraturas que se enquadram nos critérios de Lafontaine após redução incruenta. ${ }^{7}$ Entre as opções para o tratamento cirúrgico estão a fixação percutânea com fios de Kirschner (FKs), uso de placas volar ou dorsal, e fixador externo. ${ }^{8}$ Os métodos mais difundidos são FKs e placa volar devido ao fácil acesso a esses materiais. Porém, em fraturas muito cominutas em traumas de grande energia ou por ossos osteoporóticos, esses métodos podem não ter o desfecho adequado. ${ }^{1,3}$ Frente à dificuldade de estabilização dessas fraturas articulares, foi necessário procurar novas formas de fixação. Outras opções são o uso de fixador externo e placa ponte associados ao FK. ${ }^{9}$ O fixador externo é de rápida colocação, com menor agressão à pele, mas é esteticamente pior para os pacientes, e com maior risco de infecção no trajeto de pinos. ${ }^{10}$ Já a placa ponte apresenta uma maior agressão às partes moles pela incisão, mas é capaz de promover uma melhor redução da fratura. ${ }^{11}$

O presente estudo comparou os resultados pós-operatórios de fraturas cominutivas distais do rádio classificadas pelo grupo AO como $23 \mathrm{C} 2$ ou $23 \mathrm{C} 3$ tratadas cirurgicamente com fixador externo associado com FKs ou com placa ponte dorsal.

\section{Materiais e Métodos}

\section{Ética}

Este foi um estudo observacional, analítico, o qual foi aprovado pelo Comitê de Ética e Pesquisa do Hospital Municipal São José (Joinville, SC), e aprovado sob o parecer de número 2.439.743.

\section{Participantes}

Foram selecionados 43 pacientes com diagnóstico de fraturas distais do rádio intra-articulares classificadas pela $\mathrm{AO}$ como 23C2 ou C3, e com pelo menos 1 ano de pós-operatório em acompanhamento ambulatorial. Deste total 18 pacientes foram tratados com fixador externo, e 25, com placa ponte, associados ao uso de FKs, quando necessário. O tamanho da amostra foi baseado em estudos previamente publicados que compararam técnicas cirúrgicas de fraturas distais do rádio utilizando o questionário Disabilities of the Arm, Shoulder and Hand (DASH). ${ }^{12,13}$ 


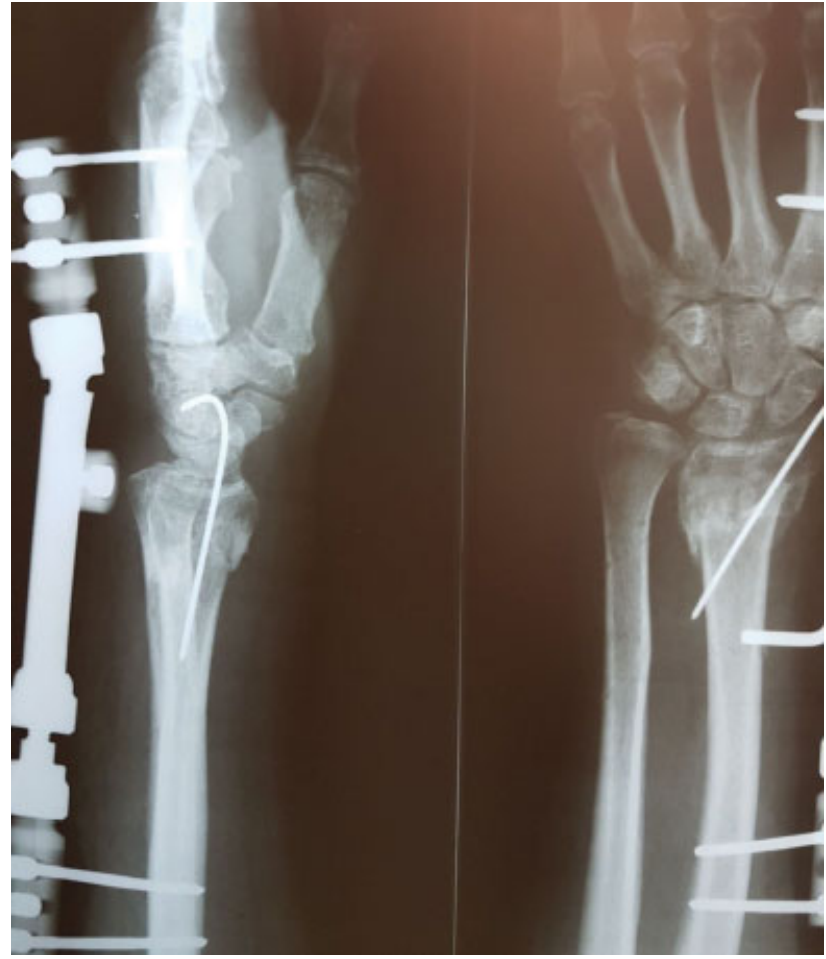

Fig. 1 Radiografia do pós-operatório com fixador externo.

O recrutamento dos pacientes aconteceu no período de dezembro de 2017 a maio de 2018 durante os retornos ambulatoriais no Hospital Municipal São José (Joinville, SC), nos quais foi realizado o tratamento cirúrgico, sempre pelo mesmo cirurgião de mão, chefe do serviço. Foram excluídos do estudo pacientes com fraturas associadas no mesmo membro; fraturas distais do rádio expostas; fratura e/ou deformidades prévias; e paciente com necessidade de internação em unidade de terapia intensiva.

\section{Técnicas Cirúrgicas}

Nos pacientes tratados com fixador externo, foi realizada a colocação dos pinos de Schanz na diáfise do segundo metacarpo e na diáfise do rádio com o auxílio da fluoroscopia,a redução da fratura, e a fixação com o fixador externo de punho tipo Colles. Quando necessário, foi associado o uso de FK para manter a redução e adicionar estabilidade (-Figura 1). No acompanhamento pós-operatório de seis a oito semanas, o fixador externo e os FKs foram retirados com anestesia local nos pinos de Schanz, conforme avaliação radiográfica. Em seguida, o paciente foi encaminhado para fisioterapia e reabilitação.

A técnica de placa ponte dorsal foi descrita inicialmente como forma de distração em fraturas mais complexas do tipo C3; porém, neste trabalho, foi utilizada como alternativa às fraturas do tipo $\mathrm{C} 2$, que são mais comuns nos atendimentos hospitalares.

Para os pacientes tratados com placa ponte dorsal e parafusos, foi realizada uma incisão em região de diáfise de terceiro metacarpo de aproximadamente $2 \mathrm{~cm}$, uma incisão em região de terceiro túnel extensor no nível do tubérculo de Lister, para isolar o tendão extensor longo do polegar, e uma na diáfise do rádio (-Figura 2), e a placa é colocada de forma retrógrada.

A placa de compressão dinâmica (PCD) foi deslizada justa óssea, afastando o tendão extensor longo do polegar no terceiro túnel por um acesso de aproximadamente $3 \mathrm{~cm}$ no tubérculo de Lister. A placa é passada abaixo dos túneis extensores, e o acesso é realizado para isolar o tendão do extensor longo do polegar e se certificar de que a placa está abaixo dos tendões. Foi realizada a fixação com dois parafusos

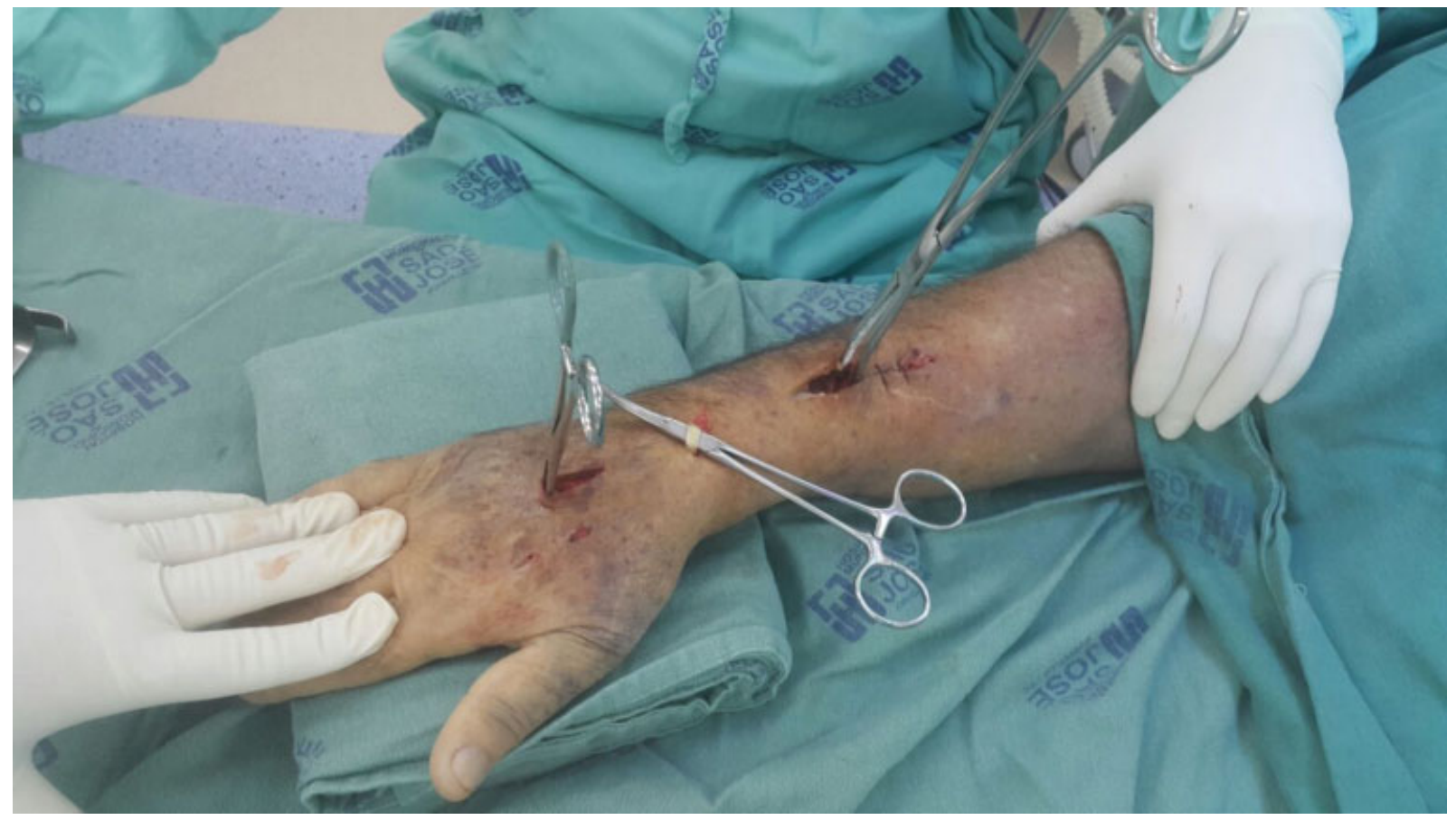

Fig. 2 Intraoperatório pela técnica de placa ponte. 


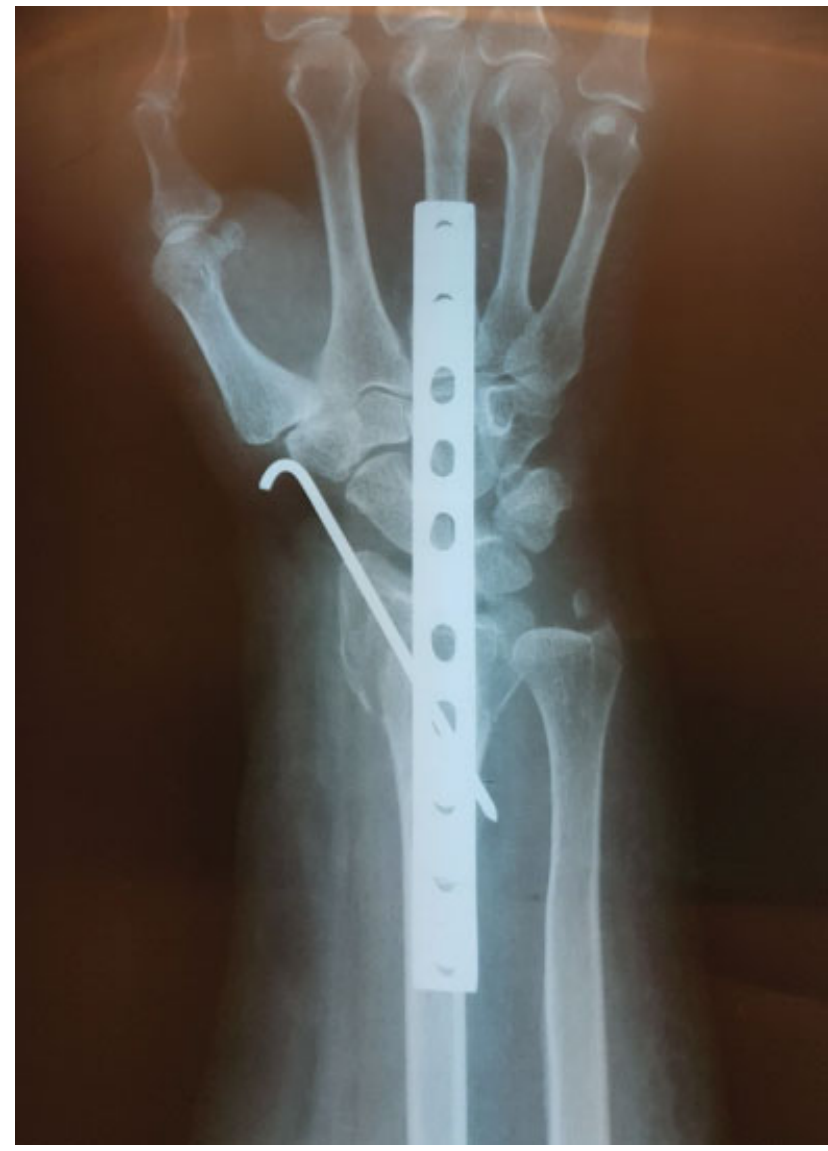

Fig. 3 Radiografia do pós-operatório de placa dorsal.

distais, a redução da fratura, e, depois, a fixação com dois parafusos proximais. Se necessário, complementou-se a síntese com FKs (-Figura 3).

Em ambas as técnicas, foi utilizado antibiótico intraoperatório (cefazolina) e antibiótico profilático por via oral (cefalexina) por 10 dias.

Foi padronizado o primeiro retorno em duas semanas após o procedimento cirúrgico para a remoção da sutura e orientações gerais. Após seis semanas da cirurgia, avaliamos a mobilidade dos dedos e retiramos o fixador externo e os FKs. Após 12 semanas da cirurgia, avaliamos a mobilidade dos dedos dos pacientes que retiraram o fixador externo, e foi retirado o material de síntese em centro cirúrgico sob anestesia, e realizada a manipulação articular. Após a retirada do material de síntese, em ambos os grupos, os pacientes foram encaminhados para fisioterapia e reabilitação, retornando a cada 12 semanas para avaliação da mobilidade, até completar 1 ano de acompanhamento ambulatorial.

\section{Variáveis Avaliadas}

As variáveis de desfecho foram coletadas após um ano do procedimento cirúrgico e de acompanhamento ambulatorial. A presença de infecção superficial ou profunda e a incidência de neuropatia simpático-reflexa foram detectadas por meio da análise dos prontuários médicos, pois o diagnóstico é clínico em ambas as situações.

Utilizamos a escala visual analógica (EVA) para a avaliação da dor pós-operatória.
Para a avaliação funcional dos pacientes, aplicamos o questionário DASH, que é composto por trinta perguntas relacionadas às limitações dos pacientes para a realização de suas atividades de vida diária. ${ }^{14} \mathrm{O}$ DASH foi aplicado sempre pelo mesmo avaliador. As questões eram lidas e explicadas antes de o paciente respondê-las.

As medidas radiográficas para analisar os ângulos do rádio distal foram feitas em radiografias anteroposteriores (APs) e de perfil com a técnica adequada, com o auxílio de um goniômetro.

A amplitude de movimento foi avaliada pelo exame físico, com valores obtidos com o goniômetro nas posições de flexão (normal: $70^{\circ}$ a $80^{\circ}$ ), extensão (normal: $60^{\circ}$ a $70^{\circ}$ ), pronação máxima (normal: $0^{\circ}$ a $80^{\circ}$ ), supinação máxima (normal: $0^{\circ}$ a $90^{\circ}$ ), desvio radial (normal: $20^{\circ}$ ), e desvio ulnar (normal: $\left.45^{\circ}\right) .^{15}$

A força de preensão foi verificada com um dinamômetro digital calibrado, sempre pelo mesmo operador, sendo comparados os valores dos pacientes em ambas as mãos, com um lado sendo considerado normal, diminuído ou aumentado em relação ao lado oposto.

\section{Análises Estatísticas}

Os dados foram tabulados em planilha do programa Excel (Microsoft Corp. Redmond, WA, EUA) e submetidos a análise estatística por meio do programa Statistical Package for the Social Sciences (SPSS, IBM Corp., Armonk, NY, EUA), versão 20.0. Foram realizadas análises de frequência e distribuição das variáveis. As variáveis nominais foram relacionadas às técnicas cirúrgicas por meio do teste qui-quadrado. As variáveis numéricas foram relacionadas às técnicas cirúrgicas por meio do teste não-paramétrico de Mann-Whitney.

\section{Resultados}

Dos 43 pacientes avaliados, a média de idade foi de 54,4 anos, sendo 25 pacientes $(58,1 \%)$ do gênero feminino. 0 mecanismo de trauma mais prevalente foi a queda do mesmo nível (22 casos; 51,2\%). Em 22 pacientes (51,2\%), a fratura ocorreu do lado direito, e em 34 pacientes (79,1\%), o lado dominante era o direito ( Tabela $\mathbf{1}$ ).

Quanto à presença de infecção pós-operatória, constatamos um caso na técnica de placa ponte e dois casos na técnica de fixador externo, não havendo diferença significativa entre as duas técnicas ( $p=0,379$ ). A neuropatia simpático-reflexa ocorreu em dois pacientes submetidos à técnica de placa ponte, e em um paciente submetido à técnica com fixador externo, também não havendo diferença estatisticamente significativa entre as duas técnicas $(p=0,738)$. Quanto à força de preensão, 15 pacientes operados com placa ponte apresentaram força diminuída, enquanto apenas 6 pacientes com fixador externo apresentaram essa alteração, havendo diferença estatisticamente significativa entre as duas técnicas cirúrgicas ( $p=0,041)$ (-Tabela 2 ).

De acordo com a EVA, ambas as técnicas apresentaram o mesmo nível de dor, não havendo diferença estatisticamente significativa entre elas. A média do escore do DASH para os pacientes operados com placa ponte foi de 39,05, e a dos 
Tabela 1 Análise descritiva das características demográficas, cirurgias e causas das fraturas

\begin{tabular}{|c|c|c|}
\hline \multicolumn{2}{|c|}{ Características } & \multirow[t]{2}{*}{ N (\%) } \\
\hline Gênero & & \\
\hline & Masculino & $18(41,9)$ \\
\hline & Feminino & $25(58,1)$ \\
\hline \multicolumn{3}{|c|}{ Idade (anos) } \\
\hline & $20-35$ & $9(20,9)$ \\
\hline & $36-45$ & $5(11,6)$ \\
\hline & $46-60$ & $9(20,9)$ \\
\hline & $>61$ & $20(46,5)$ \\
\hline \multicolumn{3}{|c|}{ Técnica cirúrgica } \\
\hline & Placa ponte & $25(58,2)$ \\
\hline & Fixador externo & $18(41,8)$ \\
\hline \multicolumn{3}{|c|}{$\begin{array}{l}\text { Mecanismo } \\
\text { do trauma }\end{array}$} \\
\hline & Queda do mesmo nível & $22(51,2)$ \\
\hline & Queda de altura & $14(31,7)$ \\
\hline & Acidente automobilístico & $7(17,1)$ \\
\hline \multicolumn{3}{|c|}{ Lado da fratura } \\
\hline & Direito & $22(51,2)$ \\
\hline & Esquerdo & $21(48,8)$ \\
\hline \multicolumn{3}{|c|}{ Lado dominante } \\
\hline & Direito & $34(79,1)$ \\
\hline & Esquerdo & $9(20,9)$ \\
\hline
\end{tabular}

Tabela 2 Análise comparativa entre as duas técnicas cirúrgicas

\begin{tabular}{|l|l|l|l|l|}
\hline \multicolumn{2}{|l|}{ Técnicas cirúrgicas } & $\begin{array}{l}\text { Placa } \\
\text { ponte }\end{array}$ & $\begin{array}{l}\text { Fixador } \\
\text { externo }\end{array}$ & $\begin{array}{l}\text { Valor } \\
\text { de } p\end{array}$ \\
\hline \multirow{2}{|c|}{} & Sim & 1 & 2 & 0,379 \\
\cline { 2 - 4 } $\begin{array}{l}\text { Infecção } \\
\text { pós-operatória }\end{array}$ & Não & 24 & 16 & \\
\hline $\begin{array}{l}\text { Neuropatia } \\
\text { simpático-reflexa }\end{array}$ & Sim & 2 & 1 & 0,738 \\
\cline { 2 - 4 } & Não & 23 & 17 & \\
\hline \multirow{2}{*}{$\begin{array}{l}\text { Força de } \\
\text { preensão }\end{array}$} & Normal & 10 & 12 & $\mathbf{0}, 041$ \\
\cline { 2 - 4 } & Diminuída & 15 & 6 & \\
\hline
\end{tabular}

operados com fixador externo foi de 36,36 , não havendo diferença estatisticamente significativa entre elas $(p=0,569)$. Quanto às medidas radiográficas, também não foram encontradas diferenças estatisticamente significativas entre as duas técnicas cirúrgicas em nenhuma das medidas realizadas. Quanto às amplitudes de movimento de extensão, flexão, pronação e desvio radial, também não foi encontrada diferença estatisticamente significativa. Na supinação, a média foi de 41,82 e 59,29 para placa ponte e fixador externo, respectivamente, com diferença estatisticamente significativa $(p=0,012)$. Na média do desvio ulnar também houve diferença estatistica-
Tabela 3 Médias e desvios padrão para as duas técnicas cirúrgicas, considerando as variáveis numéricas

\begin{tabular}{|l|l|l|l|}
\hline & Placa ponte & $\begin{array}{l}\text { Fixador } \\
\text { externo }\end{array}$ & $\begin{array}{l}\text { Valor } \\
\text { de } \boldsymbol{p}\end{array}$ \\
\hline Escala de dor & $3,68 \pm 2,61$ & $4,64 \pm 2,43$ & 0,172 \\
\hline DASH & $39,05 \pm 13,75$ & $36,36 \pm 12,03$ & 0,569 \\
\hline $\begin{array}{l}\text { Medidas } \\
\text { radiográficas }\end{array}$ & & & \\
\hline Altura radial & $8,14 \pm 2,27$ & $9,29 \pm 2,19$ & 0,069 \\
\hline Ângulo radial & $15,91 \pm 4,45$ & $17,86 \pm 3,18$ & 0,217 \\
\hline Desvio volar & $3,55 \pm 4,00$ & $4,21 \pm 3,66$ & 0,329 \\
\hline $\begin{array}{l}\text { Amplitude de } \\
\text { movimento } \\
\text { (ADM) }\end{array}$ & & & \\
\hline ADM - extensão & $40,45 \pm 16,75$ & $45,71 \pm 14,39$ & 0,667 \\
\hline ADM - flexão & $51,14 \pm 16,68$ & $52,14 \pm 18,47$ & 0,438 \\
\hline ADM - pronação & $43,18 \pm 18,09$ & $58,21 \pm 17,71$ & 0,062 \\
\hline ADM - supinação & $41,82 \pm 18,67$ & $59,29 \pm 20,17$ & $\mathbf{0 , 0 1 2}$ \\
\hline $\begin{array}{l}\text { ADM - desvio } \\
\text { ulnar }\end{array}$ & $7,73 \pm 7,02$ & $13,57 \pm 8,41$ & $\mathbf{0 , 0 0 4 9}$ \\
\hline $\begin{array}{l}\text { ADM - desvio } \\
\text { radial }\end{array}$ & $7,73 \pm 6,31$ & $12,86 \pm 6,41$ & 0,066 \\
\hline
\end{tabular}

Abreviatura: DASH, Disabilities of Arm, Shoulder and Hand.

mente significativa entre as duas técnicas cirúrgicas ( $p=0,0049$ ), sendo os valores de 7,73 para a placa ponte e de 13,57 para o fixador externo (-Tabela $\mathbf{3}$ ).

\section{Discussão}

Pelo que sabemos, existem poucos estudos comparando a fixação de fraturas cominutivas com fixador externo e placa ponte. Essas fraturas são muito prevalentes e de difícil manejo, e não existe uma técnica cirúrgica que seja consenso quanto ao manejo. Nesse panorama, o presente trabalho foi realizado para mostrar os resultados com materiais de fácil acesso no Sistema Único de Saúde (SUS), e que podem proporcionar uma sobrevida melhor ao paciente.

O uso da técnica de placa ponte dorsal é indicada nos casos de fratura articular, cominutiva, desviada, com fragmentos muito pequenos que não podem ser fixados por placa volar. ${ }^{16}$ Alguns autores ${ }^{16,17}$ defendem o uso de placa dorsal pela ligamentotaxia, que auxilia na redução da fratura, bem como pela manutenção das partes moles. E o fato de os tendões dos extensores ficarem sem atrito com a placa favorece uma mobilidade dos dedos, com o seu uso sendo indicado em ossos osteopênicos, fraturas cominutivas, e politrauma. ${ }^{17}$ No entanto, a placa dorsal favorece a perda da mobilidade do punho, a necessidade de sua remoção, e a redução da fratura em apenas um plano. ${ }^{17}$ Além da PCD de 3,5 mm, temos disponibilidade das placas terço de cana em coluna única no rádio ou para dupla coluna. Quando o traço da fratura é mais proximal, temos como opção as placas de reconstrução de $2,4 \mathrm{~mm}$ e $3,5 \mathrm{~mm}$, que têm maior disponibilidade no SUS. Outros tipos de placas foram desenvolvidos para essa técnica cirúrgica, como as placas 
bloqueadas de baixo perfil, que podem ser utilizadas no segundo ou terceiro metacarpos. Placas de artrodese de punho também podem ser utilizadas para realizar a fixação das fraturas distais do rádio, sendo possível colocar parafusos maiores, tornando-as mais estáveis. Semelhante às placas de artrodese, surgiram mais recentemente placas específicas para o tratamento dessas fraturas pelo meio da distração, as placas de abrangência, cujo formato facilita a passagem pelos tendões extensores, e que têm menor quantidade de furos centrais, aumentando, assim, a rigidez. ${ }^{16,18}$

O uso do fixador externo transarticular de punho teve seu início em $1970,{ }^{17}$ e tem sido cada vez mais frequente. Tem como vantagem ser minimamente invasivo, não acessando o retináculo e os tendões, além de ser uma técnica fácil e rápida de ser utilizada no politraumatizado. ${ }^{19}$

Comparando as duas técnicas cirúrgicas no presente estudo, de uma maneira geral, não observamos diferenças estatisticamente significativas entre elas, o que também foi constatado por Saving et al., ${ }^{20}$ que avaliaram o pós-operatório com fixador externo e placa volar com três anos de evolução.

Considerando as complicações, observamos três casos de infecção pós-operatória, dois com fixador externo e um pela placa ponte. Um dos casos de infecção com fixador externo foi superficial e tratado com antibiótico por via oral; no outro, foi necessário debridamento cirúrgico e realinhamento do fixador. Na infecção da placa ponte, foi necessário debridamento cirúrgico com antibioticoterapia endovenosa. Cui et al. ${ }^{21} \mathrm{com}-$ pararam o uso de síntese interna versus externa em pacientes com fratura distal do rádio $\mathrm{AOC} 3$, e observaram maior taxa de infecção com o uso de síntese externa. Abramo et al. ${ }^{22}$ avaliaram 50 pacientes com fratura distal do rádio instável com as técnicas de redução aberta e fixação interna e de redução fechada e fixação externa, e observaram equivalência nas taxas de infecções superficiais e profundas em ambas as técnicas, corroborando nossos achados. A principal complicação pósoperatória em seu estudo ${ }^{22}$ foi hiperestesia em trajeto de nervo sensitivo radial. Kreder et al. ${ }^{23}$ avaliaram 179 pacientes com fratura distal do rádio tratados com fixação percutânea e fixação interna, sendo utilizados fixadores externos e placas. A infecção foi a complicação pós-operatória mais comum, sendo que a infecção superficial ocorreu mais na redução indireta com fixação percutânea, e a infecção profunda foi semelhante em ambos os grupos. Em um estudo de metanálise ${ }^{18}$ que comparou fixador externo com placa ponte, os autores encontraram menor incidência de infecção e de distrofia simpáticoreflexa nos pacientes tratados com placa ponte, mas os casos de distrofia não necessitaram de tratamento cirúrgico adicional. $^{18}$

A neuropatia simpático-reflexa ocorreu em dois pacientes com placa e em um paciente com fixador externo, correspondendo a quase $7 \%$ do total dos pacientes, e todos eram do sexo feminino. Xu et al. ${ }^{24}$ compararam a fixação de fraturas do tipo $C$ da classificação AO com fixação externa e com placa, e não observaram nenhum caso de distrofia simpáticoreflexa. Arora et al. ${ }^{25}$ avaliaram a incidência de neuropatia simpático-reflexa em pacientes acima de 65 anos com fratura de distal do rádio, com 36 pacientes submetidos a cirurgia e 37 pacientes submetidos a tratamento conserva- dor. Dois pacientes submetidos a cirurgia e cinco com tratamento conservador desenvolveram a síndrome. Todos os pacientes tiveram melhora do quadro com analgesia e fisioterapia. Xavier et al., ${ }^{26}$ em seu estudo, avaliaram pacientes tratados com placa volar bloqueada em fraturas distais do rádio, e encontraram a incidência de 1,5\% de distrofia simpático-reflexa, o que mostra que até com materiais mais modernos essa complicação pode ocorrer.

A força de preensão teve um resultado melhor com o uso de fixador externo. Isso provavelmente aconteceu pela mobilidade dos dedos conferida mais precocemente pelo não uso de imobilização gessada, mantendo-se em movimento já no pós-operatório imediato, e, também, pela menor manipulação da musculatura que ocorre no acesso cirúrgico para a placa dorsal. Este achado vai de encontro com o obtido por Schønnemann et al., ${ }^{27}$ que observaram resultados melhores nos pacientes com material de síntese interno em comparação à fixação externa com a intramedular. Aita et al. ${ }^{28}$ compararam a fixação de rádio distal com placa, haste e fixador externo, e observaram que, na terceira semana pósoperatória, a força de preensão com o fixador externo era menor do que nas demais técnicas, mas, após um ano, a força de preensão era similar entre as três técnicas.

McQueen e Caspers ${ }^{29}$ observaram que as principais complicações de fraturas distais do rádio são dor, rigidez, deformidade, e perda da força de preensão. Esses achados se mantêm até os dias atuais, apesar dos grandes avanços nas técnicas cirúrgicas.

No presente estudo, observamos que, independente do método, a dor pós-operatória após a retirada do material de síntese e a realização de fisioterapia foi semelhante em ambas as técnicas cirúrgicas. Poderíamos atribuir isso à redução das fraturas com dados radiográficos semelhantes, pois, com uma melhor redução da fratura, a amplitude de movimento é melhor e com menos dor. ${ }^{30}$ Aita et al. ${ }^{28}$ compararam as técnicas de placa volar bloqueada, haste intramedular e fixador externo em fraturas distais do rádio, e constataram menor taxa de dor em pacientes com material de síntese interno do que em fixadores externos após a terceira semana.

Os escores do DASH foram semelhantes para ambas as técnicas cirúrgicas. Esses dados também foram obtidos no estudo de Zenke et al., ${ }^{31}$ que não mostrou diferença significativa na comparação entre as técnicas. Xavier et al. ${ }^{26}$ encontraram em seu estudo uma relação entre a perda de extensão, a flexão e a força de preensão com piores resultados do DASH, com um valor de DASH médio de 10 para jovens e de 21 para idosos.

$\mathrm{Na}$ avaliação radiográfica, conseguimos uma redução aceitável das fraturas, e semelhante entre ambas as técnicas. A metanálise realizada por Cui et al. ${ }^{21}$ mostra que vários artigos que compararam as técnicas também conseguiram fazer a redução da fratura dentro dos parâmetros preconizados. Xu et al. ${ }^{24}$ encontraram diferença apenas para a inclinação radial na avaliação no decorrer dos anos, sem alterar a amplitude de movimento do paciente.

A amplitude de movimento encontrado foi similar entre as duas técnicas no nosso estudo, exceto para supinação e desvio ulnar, que tiveram resultados melhores com uso do fixador externo. Apesar dessa diferença, em ambas as 
técnicas os pacientes atingiram amplitudes de movimento funcionais. Xavier et al. ${ }^{26}$ também não encontraram diferença em relação à amplitude de movimento dos pacientes. Kreder et al. ${ }^{23}$ tampouco observaram diferenças entre os diferentes grupos na mobilidade pós-operatória. Richard et al. $^{32}$ avaliaram 33 pacientes com mais de 60 anos, com ossos osteoporóticos, com fraturas classificadas como C2 ou C3, que foram tratados pela técnica de placa ponte dorsal, demonstrando bons resultados para consolidação e amplitude de movimento.

No estudo de Lewis et al., ${ }^{33}$ avaliou-se a incidência de complicações nas fixações no segundo ou terceiro metacarpos em cadáveres. Os autores concluíram que a fixação no terceiro metacarpo tem uma chance maior de lesão do extensor longo do polegar, e que a flexo-extensão do punho foi um indicador ruim.

As constatações do presente estudo são de grande importância para o progresso das fixações de fraturas distais do rádio graves, pois os pacientes necessitam retornar às suas atividades diárias e profissionais o mais breve possível. As duas técnicas operatórias são de fácil execução para cirurgiões ortopedistas, e com material barato e de fácil acesso nos serviços públicos.

Quanto às limitações deste estudo, poderíamos considerar o número reduzido de pacientes avaliados e a idade com amplo desvio padrão, com diferentes qualidades ósseas. Uma sugestão para um estudo futuro seria aumentar o número de pacientes, fazer uma estratificação por idade, e incluir outros métodos de fixação disponíveis, como a haste intramedular de rádio e a placa bloqueada de rádio distal.

\section{Conclusão}

Ambas as técnicas se mostraram eficazes para o tratamento de fraturas complexas distais do rádio, com um baixo índice de complicações pós-operatórias e com resultados funcionais satisfatórios, não sendo possível afirmar a superioridade de uma em relação à outra.

Conflito de Interesses

Os autores declaram não haver conflito de interesses.

\section{Referências}

1 Alffram PA, Bauer GC. Epidemiology of fractures of the forearm. A biomechanical investigation of bone strength. J Bone Joint Surg Am 1962;44-A:105-114

2 Arora R, Gabl M, Gschwentner M, Deml C, Krappinger D, Lutz M. A comparative study of clinical and radiologic outcomes of unstable colles type distal radius fractures in patients older than 70 years: nonoperative treatment versus volar locking plating. J Orthop Trauma 2009;23(04):237-242

3 Tumia N, Wardlaw D, Hallett J, Deutman R, Mattsson SA, Sandén B. Aberdeen Colles' fracture brace as a treatment for Colles' fracture. A multicentre, prospective, randomised, controlled trial. J Bone Joint Surg Br 2003;85(01):78-82

4 Glickel SZ, Catalano LW, Raia FJ, Barron OA, Grabow R, Chia B. Long-term outcomes of closed reduction and percutaneous pinning for the treatment of distal radius fractures. J Hand Surg Am 2008;33(10):1700-1705
5 Lee DY, Park YJ, Park JS. A Meta-analysis of studies of volar locking plate fixation of distal radius fractures: conventional versus minimally invasive plate osteosynthesis. Clin Orthop Surg 2019;11(02):208-219

6 Handoll HHG, Madhok R. Closed reduction methods for treating distal radial fractures in adults. Cochrane Database Syst Rev 2003; (01):CD003763

7 LaMartina J, Jawa A, Stucken C, Merlin G, Tornetta P III. Predicting alignment after closed reduction and casting of distal radius fractures. J Hand Surg Am 2015;40(05):934-939

8 Piva Neto A, Lhamby FC. Fixação das fraturas da extremidade do rádio distal pela técnica de Kapandji: avaliação dos resultados radiológicos. Rev Bras Ortop 2011;46(04):368-373

9 Snow M, Kelly M, Jeyam M, Fahmy N. Functional versus position: a randomized controlled trial of interfocal Kirschner wiring of unstable distal radial fractures. Eur J Trauma Emerg Surg 2007; 33(01):81-86

10 Benson LS, Minihane KP, Stern LD, Eller E, Seshadri R. The outcome of intra-articular distal radius fractures treated with fragmentspecific fixation. J Hand Surg Am 2006;31(08):1333-1339

11 Azad A, Intravia JM, Hill JR, et al. Carpal translocation following dorsal bridge plate fixation of distal radius fractures: a cadaveric study. J Wrist Surg 2019;8(03):234-239

12 Harley BJ, Scharfenberger A, Beaupre LA, Jomha N, Weber DW. Augmented external fixation versus percutaneous pinning and casting for unstable fractures of the distal radius-a prospective randomized trial. J Hand Surg Am 2004;29(05):815-824

13 Atroshi I, Brogren E, Larsson GU, Kloow J, Hofer M, Berggren AM. Wrist-bridging versus non-bridging external fixation for displaced distal radius fractures: a randomized assessor-blind clinical trial of 38 patients followed for 1 year. Acta Orthop 2006;77(03):445-453

14 Hudak PL, Amadio PC, Bombardier C; The Upper Extremity Collaborative Group (UECG). Development of an upper extremity outcome measure: the DASH (disabilities of the arm, shoulder and hand) [corrected]. Am J Ind Med 1996;29(06):602-608

15 Barros Filho TEP, Lech O. Exame físico em ortopedia. 3å . Ed. São Paulo: Editora Sarvier; 2017

16 Vakhshori V, Alluri RK, Stevanovic M, Ghiassi A. Review of internal radiocarpal distraction plating for distal radius fracture fixation. Hand (N Y) 2018:1558944718787877

17 Hyatt BT, Hanel DP, Saucedo JM. Bridge plating for distal radius fractures in low-demand patients with assist devices. J Hand Surg Am 2019;44(06):507-513

18 Tinsley BA, Ilyas AM. Distal radius fractures in a functional quadruped: spanning bridge plate fixation of the wrist. Hand Clin 2018;34(01):113-120

19 Mishra AK, Sud AD, Prasad M, Kaul R, Singh CM. A comparative study of functional outcome of external fixation and volar plating in unstable distal radius fractures. Int J Res Orthop 2019;5(02): 326-334

20 Saving J, Enocson A, Ponzer S, Mellstrand Navarro C. External Fixation Versus Volar Locking Plate for Unstable Dorsally Displaced Distal Radius Fractures-A 3-Year Follow-Up of a Randomized Controlled Study. J Hand Surg Am 2019;44(01):18-26

21 Cui Z, Pan J, Yu B, Zhang K, Xiong X. Internal versus external fixation for unstable distal radius fractures: an up-to-date metaanalysis. Int Orthop 2011;35(09):1333-1341

22 Abramo A, Kopylov P, Geijer M, Tägil M. Open reduction and internal fixation compared to closed reduction and external fixation in distal radial fractures: a randomized study of 50 patients. Acta Orthop 2009;80(04):478-485

23 Kreder HJ, Hanel DP, Agel J, et al. Indirect reduction and percutaneous fixation versus open reduction and internal fixation for displaced intra-articular fractures of the distal radius: a randomised, controlled trial. J Bone Joint Surg Br 2005;87(06):829-836

24 Xu GG, Chan SP, Puhaindran ME, Chew WY. Prospective randomised study of intra-articular fractures of the distal radius: 
68 Análise clínica e radiológica do resultado placa ponte Carula et al.

comparison between external fixation and plate fixation. Ann Acad Med Singapore 2009;38(07):600-606

25 Arora R, Lutz M, Deml C, Krappinger D, Haug L, Gabl M. A prospective randomized trial comparing nonoperative treatment with volar locking plate fixation for displaced and unstable distal radial fractures in patients sixty-five years of age and older. J Bone Joint Surg Am 2011;93(23):2146-2153

26 Xavier CR, Molin DC, Santos RM, Santos RD, Ferreira Neto JC. Tratamento cirúrgico das fraturas do radio distal com placa volar bloqueada: correlação dos resultados clínicos e radiográficos. Rev Bras Ortop 2011;46(05):505-523

27 Schønnemann JO, Hansen TB, Søballe K. Randomised study of non-bridging external fixation compared with intramedullary fixation of unstable distal radial fractures. J Plast Surg Hand Surg 2011;45(4-5):232-237

28 Aita MA, Ferreira CH, Ibanez DS, et al. Ensaio clínico randomizado de osteossíntese percutânea e minimamente invasiva das fraturas da extremidade distal do rádio. Rev Bras Ortop 2014;49(03):218-226
29 McQueen M, Caspers J. Colles fracture: does the anatomical result affect the final function? J Bone Joint Surg Br 1988;70(04): 649-651

30 Andrade LE, Oliveira D, Barros JW. Tratamento das fraturas instáveis da extremidade distal do rádio com desvio volar utilizando-se placa de apoio. Acta Ortop Bras 2002;10(04):5-9

31 Zenke Y, Sakai A, Oshige T, et al. Clinical results of volar locking plate for distal radius fractures: conventional versus minimally invasive plate osteosynthesis. J Orthop Trauma 2011;25(07): 425-431

32 Richard MJ, Katolik LI, Hanel DP, Wartinbee DA, Ruch DS. Distraction plating for the treatment of highly comminuted distal radius fractures in elderly patients. J Hand Surg Am 2012;37(05): 948-956

33 Lewis S, Mostofi A, Stevanovic M, Ghiassi A. Risk of tendon entrapment under a dorsal bridge plate in a distal radius fracture model. J Hand Surg Am 2015;40(03):500-504 